\title{
Al Applications to Distribution System with Distributed Generation
}

\author{
Dr.P.N.Hrisheekesha \\ Associate Professor Department of \\ $E \& E$ \\ Manipal Institute of Technology \\ Manipal University, Manipal
}

\author{
Dr. Jaydev Sharma \\ Professor \\ Department of Electrical Engg., \\ Indian Institute of Technology Roorkee
}

\begin{abstract}
In this paper, Artificial Neural Network, one of the Artificial Intelligence (AI) techniques, for the Volt / Var control in power distribution systems with dispersed generation (DG) is proposed. Artificial neural networks have been considered due to their ability for real time control, simpler calculations and adaptability to different operating conditions. Neuro-controllers are much more effective, fast acting than conventional controllers. Neural network for controlling Step voltage regulator (SVR) with line rise compensation (LRC) /line drop compensation (LDC) function has been presented. The neural network based controller has been simulated for a radial distribution system with DG and the neurocontroller shows promising results after testing.
\end{abstract}

\section{Keywords}

Artificial Intelligence, Artificial neural network, Dispersed generation, Distribution system, Line drop compensation, Line rise compensation, Step Voltage regulator, Voltage / Reactive power control.

\section{INTRODUCTION}

The ever increasing demand of electricity and increased concerned over environmental issues, has posed many challenges on power system companies and in a way distributed generation (DG) has emerged as one of the most suitable alternatives to solve these issues. The distributed generation means embedding of small generators in the capacity ranging from $15 \mathrm{~kW}$ to $20 \mathrm{MW}$ (even up to $50 \mathrm{MW}$ ) in a distribution system which are scattered throughout a power system to provide electric power needed by consumers, thereby displacing the need to build additional or upgrade local distribution lines as well as improving the system performance.

In the deregulated power system environment, volt/var control in the distribution system embedded with DGs has become a key issue for the distribution system planning, operation and automation. Distributed generation can have significant impact on voltages, load demand, power losses, system reliability and economy in distribution networks. Introduction of distributed generation causes reverse power flows and complicated voltage profiles in the distribution systems [1], [2]. When the voltages at different buses are not within the statutory limits, ANSI C84.11995, proper measures must be taken to improve the voltage profile [1]-[4].
Since conventional voltage regulators cannot handle the reverse power flow and steep voltage fluctuations, new voltage regulation methods are required to be adopted to solve these problems. The drawback of conventional controllers is that once the operating point or the system configuration changes, the performance of the controller degrades. Neuro controllers ensure superior response throughout the system, for different operating conditions compared to conventional controllers [5], [12].

Since volt/var control is directly related to the power quality of the distribution network and is one of the key steps for distribution automation, it is of primary concern to consider in this research work.

In the literature different voltage control methods are discussed. Turan Gonen [6] discussed SVR with LDC voltage regulation method. T .E Kim and J.E. Kim [7] presented the voltage regulation coordination method of DGs in a power distribution system by controlling the reactive power output of the DGs. JoonHo Choi and Jae-Chul Kim [8] presented multiple line drop compensation voltage regulation method considering dispersed storage generation systems and unbalanced load diversity. TaeEung Kim and Jae-Eon Kim [9] developed correlations between the load tap changing transformer (LCT), line drop compensator (LDC) and the output of DG..

Nigel C.Scott et al [10] Proposed load control method to tackle voltage rise issues due to embedded generation. T.Niknam et al [11] proposed volt/var control in the distribution system with DG using load tap changer, capacitor and voltage regulator. Artificial Neural Network (ANN) control of capacitors and voltage regulators are reported in the literature. N.Iwan Santoso and Owen T.Tan [12] presented a two stage neural network to control multi tap capacitors in a distribution system. Yuan-Yih Hsu and Feng-Chang Lu [13] proposed controlling of capacitor status and load tap changing transformer tap positions using neural networks. K.S. Swaroop and P.S. Subhash [14] presented two stage neural network approach to control capacitor status and transformer tap in a power system.

Neural network (NN) approach for the volt/var control in a distribution system with DG by using SVR with LRC/LDC function is yet to be addressed. Towards achieving this goal and considering the limitations of conventional controllers and the merits of neuro controllers, in this paper, neural network to control SVR with LRC/LDC function for the volt/var control in a distribution system with DG has been presented. 


\section{PROBLEM FORMULATION}

It is assumed that the position of the SVR with LRC/LDC function, rating and location of DG are known and fixed for the network considered. Constant power load model is considered.

The control parameters are Resistance of LRC/LDC, Reactance of LRC/LDC and the Tap positions of the regulator

The problem is to find the tap positions of SVR, Resistance values and Reactance values of LRC/LDC for a particular loading condition and reactive power output of DG limits. While formulating the objective function, sum of the voltage deviation in all the buses and sum of the line losses and voltage and current constraints are taken into consideration. Since volt/var control has been considered, improvement of voltage profile and minimization of losses are considered to be very much significant, the voltage deviation in all the buses is minimized and the voltage is maintained within the statutory limits and the objective function is formulated as given in equation (1).

\section{Objective Function}

$\operatorname{Min}\left(W_{1} \sum_{i=1}^{n}\left|V_{i}-V_{r e f}\right|^{2}+W_{2} \sum_{j=1}^{m} \operatorname{loss}_{j}+W_{3} g[V, I]\right)$

Where,

$\mathrm{g}(\mathrm{V}, \mathrm{I})$ total violation of voltage and current constraints

$\mathrm{W}_{1}, \mathrm{~W}_{2}$, and $\mathrm{W}_{3}$ are weightage factors

\section{Constarints:}

$v_{\min }<v_{i}<v_{\max }$

Where, $v_{\min }$ is the minimum acceptable bus voltage and $v_{\max }$ is the maximum acceptable bus voltage.

$i_{\text {min }}<i_{m}<i_{\text {max }}$

Where $i_{\min }$ is the minimum acceptable line current, $i_{m}$ is the current in the $\mathrm{m}^{\text {th }}$ line and $i_{\max }$ is the maximum acceptable line current.

Since volt/ var control is considered, the improvement of voltage profile is the primary goal of this work, the objective function, minimization of the voltage deviation is given first preference and minimization of the losses is given the second preference.

Voltage limit of $\pm 5 \%$ has been considered in this study.

\section{NEURAL NETWORK BASED VOLTAGE I REACTIVE POWER CONTROL 3.1 SVR with LRC/LDC Voltage Regulation}

The introduction of DGs in to the distribution system alters the voltage profile and causes reverse power flow. Conventional voltage regulators cannot cope up with this situation and new voltage regulation methods are required to be adopted to mitigate such problems. In this work, Step Voltage Regulator with Line Rise Compensation / Line Drop Compensation function voltage regulation method has been considered.

SVRs are auto- transformers with individual taps on their windings. Most of the SVRs are designed to correct the voltage between $\pm 10 \%$ with their tap settings. LRC/LDC is a technique used to mitigate the voltage rise/ voltage drop on a bus in a distribution network there by improving the voltage profile of the distribution network with DG's connected to them or when the local load is high. This type of control is based on the line current and is achieved by the settings of adjustable resistance and reactance elements of line rise compensator/ line drop compensator.

\subsection{Neuro-Controller Architecture}

Neural networks, one of the soft computing technologies, are simplified models of the biological nervous system. ANN is an interconnected group of artificial neurons that uses a mathematical model or computational model for information processing based on a connectionist approach to computation. In most cases an ANN is an adaptive system that changes its structure based on external or internal information that flows through the network. $\mathrm{NN}$ is widely used due to its robustness, generalization, high speed and parallel information processing capabilities. A properly trained NN can be effectively used to solve unknown and untrained instances of the problem.

The neuro-controller structure designed for this work consists of a three layer feed-forward neural network with four inputs, one hidden layer and three outputs. The hidden layer has 10 hyperbolic tangent sigmoid neurons and the output has 3 linear neurons. Back propagation algorithm has been used for training this network. For the purpose of training the neural network training data has been obtained with distribution system power flow and by solving equation (1). The training of the $\mathrm{NN}$ has been carried out offline.

The inputs for the three layer NN considered for the control are bus voltage magnitudes $|\mathrm{V}|$, Active power flow $\mathrm{P}$, Reactive power flow $Q$, Reactive power output $\mathrm{Q}_{\mathrm{G}}$ and the outputs are tap positions of SVR, Resistance and Reactance setting values of LRC/LDC.

The approach of the neural-network controller for the volt/var control includes: (Figure 1) 


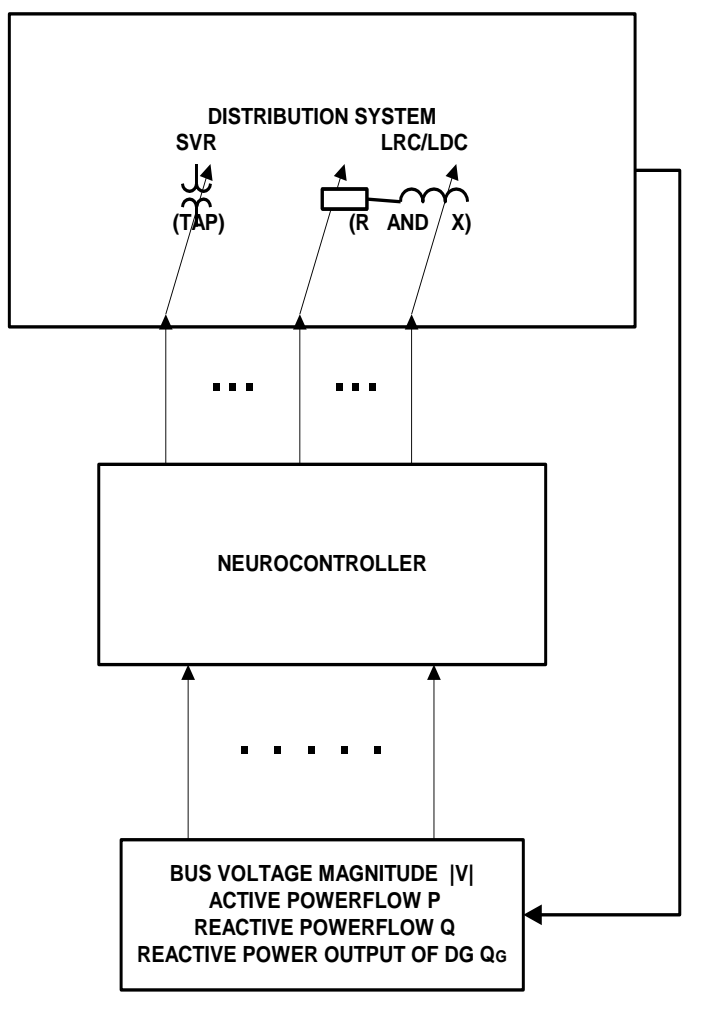

Figure 1. Neuro-controller for volt/var control

Step1. Consider the test radial distribution network with DG and equipped with SVR with LRC/LDC function.

Step2 Run the distribution power flow.

Step3 .Use the Active power flow (P), Reactive power flow (Q), bus voltage magnitude $(|\mathrm{V}|)$, Reactive power output of $\mathrm{DG}\left(\mathrm{Q}_{\mathrm{G}}\right)$ as the input to the neural network.

Step4. Train the NN with the data obtained by load flow and solving equation (1). Simulate the trained multi-layer feed forward neural network for the required operating condition.

Step5. The three outputs of neuro-controller are used to control tap position of SVR and resistance and reactance setting values of LRC/LDC. Test the NN for different on line data sets which are not used during training, so that the NN can be used for on line volt/var control of the DS with DG.

\section{TEST SYSTEM AND SIMULATION RESULTS}

\subsection{Test System}

The radial distribution system considered for this work is shown in Figure 2. [4].The parameters of the test system are shown in Table 1.
Table 1. Test System data

\begin{tabular}{ll}
\hline Network data & 5 \\
No. of buses & 4 \\
No. of branches & $1.03 \mathrm{pu}$. \\
BSP Voltage & 4 \\
No. of load buses & 1 \\
No. of DGs & Bus no.3 \\
Location of DG & \\
D G details: & $20 \mathrm{MW}$, \\
$\mathrm{P}$ & 0.9 lagging \\
Pf & $9.686 \mathrm{MVAR}$ \\
$\mathrm{Q}$ & $100 \mathrm{MVA}$ \\
Base power $\mathrm{S}_{\text {base }}$ & $1.6 \mathrm{MW}, 1.2 \mathrm{MVAR}$ \\
Load size $\mathrm{P}_{\mathrm{L}} \mathrm{Q}_{\mathrm{L}}$ & $11 \mathrm{kV}$ \\
\hline
\end{tabular}

\subsection{Simulation Results}

The test distribution system Figure 2. has been considered for the simulation of the neuro-controller. The simulation has been carried out using MATLAB.

When the DG is introduced at bus no. 3 the voltages at few buses are outside the statutory limits as shown in Figure 3.In order improve the voltage profile, one SVR at bus no.3 and LRC/LDC in the branch no. 3 has been considered. A voltage statutory limit of $\pm 5 \%$ has been considered.

For training the neural network the data has been obtained by running the distribution power flow and equation (1). For all the tap positions of SVR and different settings of $R$ and $X$ values of $\mathrm{LRC} / \mathrm{LDC}$ and for various values of $\mathrm{Q}_{\mathrm{G}}$ training data sets are obtained. One data set consists of 4 inputs and 3 output quantities. Corresponding to one particular load level about 200 data sets are obtained.

The training of NN using these data sets may not be accurate and may not generalize the network for different loading conditions. So the loading on each bus has beens divided into ten parts and training data sets are obtained for all the loading conditions. The NN has been trained with about 2000 data sets. The NN training has been done offline. The simulation results of $\mathrm{NN}$ training showed very small mean square error.

For testing the performance ability of the trained NN under different operating conditions, the $\mathrm{NN}$ is simulated for testing with different input data sets which were not used during training. The results of the neuro-controller after testing are shown in Figure 4 and are very much closer to the actual values. So the performance of the neuro-controller is found to be accurate and promising. 


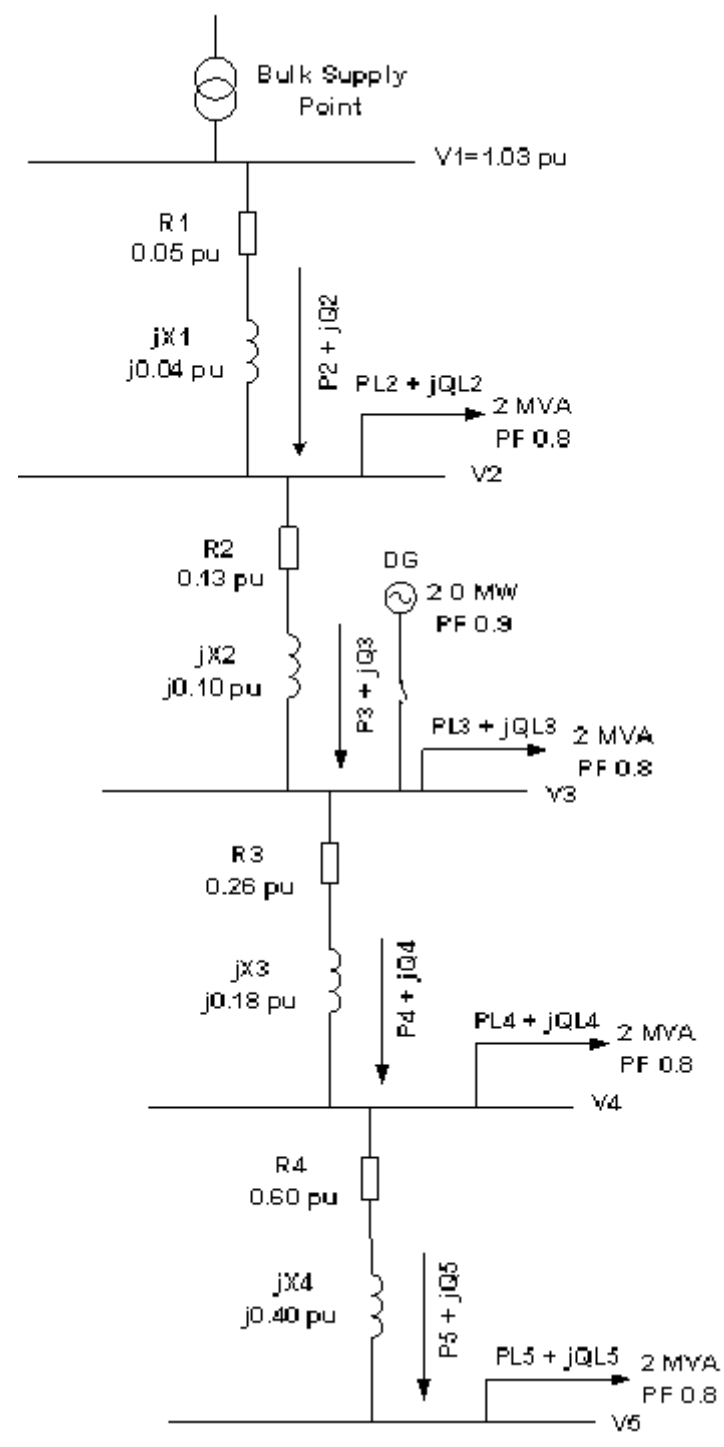

Figure 2. Test Radial Distribution Network

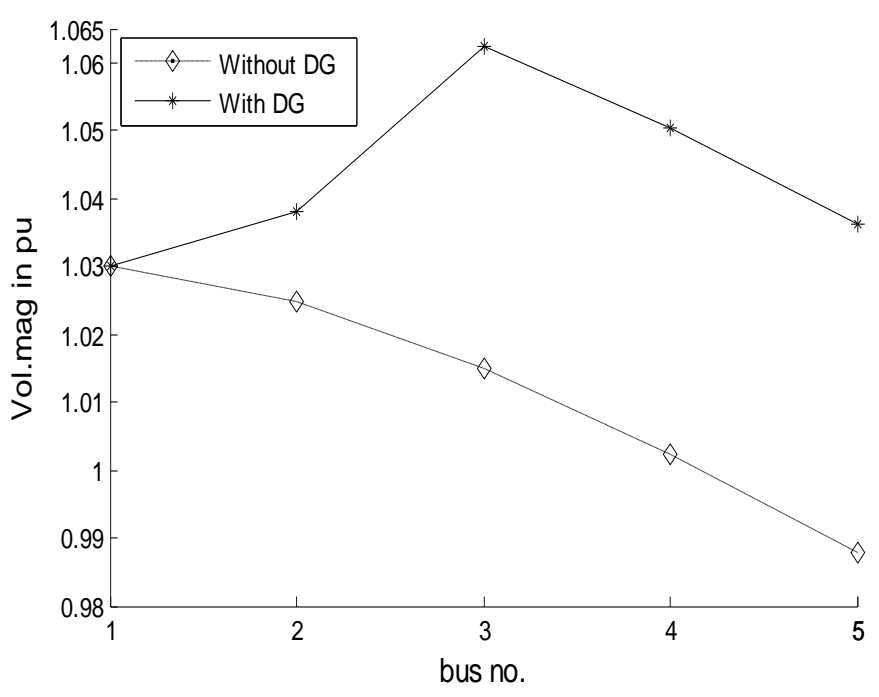

Figure 3. Voltage Profile of Test Distribution Network

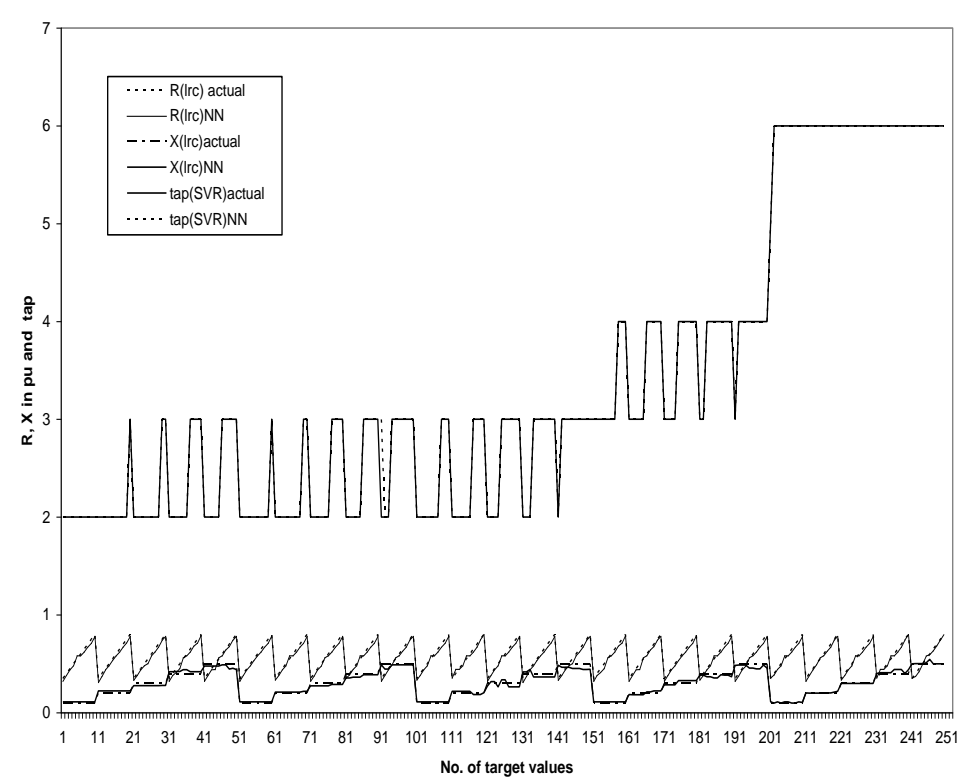

Figure 4. Neuro-Controller target outputs Vs No. of Target values

Details of legends in Fig.4.

1. Resistance of LRC/LDC actual 2. Resistance of LRC/LDC by NN 3. Reactance of LRC/LDC actual 4. Reactance of LRC/LDC by NN 5. Tap position of SVR actual position of SVR by NN. 


\section{CONCLUSIONS}

In this paper, neural network based volt/var control in radial distribution system with dispersed generation has been proposed. The neuro-controller controls the tap positions of SVR and resistance and reactance setting values of line rise compensator/line drop compensator. This neuro-controller is applicable for real-time control of SVRs with LRC/LDC functions installed in distribution systems with DGs. This controller has been developed for varying load conditions.

The results obtained by the controller are accurate. The results also illustrate that the performance of neuro-controller is better than conventional controller. The performance of a conventional controller degrades when the operating point and system configuration changes, whereas this neuro-controller shows superior response throughout the system for different operating conditions. Therefore it can be concluded this neuro-controller is effective, accurate, fast acting and suitable for real-time volt/var control in distribution system with DG. This work can become a prominent step towards distribution system automation.

\section{REFERENCES}

[1] Masters C.L.,"Voltage rise: the big issue when connecting embedded generation to long $11 \mathrm{kV}$ overhead lines", Power engineering Journal, IEE, Vol.16, No.1, pp.5-12, 2002

[2] A.E. Kiprakis and A.R Wallace, "Maximizing energy capture from distributed generators in weak networks", IEE Proceedings on Generation Transmission Distribution, Vol.151, No.5, pp.611-618, September 2004

3] "IEEE standard for interconnecting distributed resources with electric power systems" IEEE standard, $1547^{\mathrm{TM}}-2003$

[4] Khoan Tran and Mohammad Vaziri, "Effects of dispersed generation on distribution systems", IEEE conference,2005

[5] Rajshekaran S.,'Neural networks, fuzzy logic and genetic algorithms: synthesis and applications" Prentice-Hall of India, 2004

[6] Turan Gonen,'Electric power distribution system engineering" McGraw-Hill, 1986

[7] T.E. Kim and J.E.Kim, "Voltage regulation coordination of distributed generation system in distribution system", IEEE conference, pp.480-484, 2001

[8] Joon-Ho Choi and Jae-Chul Kim, "Advanced voltage regulation method at the power distribution systems interconnected with dispersed storage and generation systems", IEEE transactions on Power Delivery, Vol.15, No.2,pp.691-696, April 2000

[9] Tae-Eung Kim and Jae- Eon Kim," Considerations for the feasible operating range of distributed generation interconnected to power distribution system", IEEE Conference, pp.42-48, 2002

[10] Nigel C.Scott, David J.Atkinson and James E.Morrell,'Use of load control to regulate voltage on distribution networks with embedded generation", IEEE transactions on Power Systems, Vol.17, No.2, pp.510-515, May 2002

[11] T. Niknam, A.M. Ranjbar and A.R. Shirani, "Impact of distributed generation on Volt/ Var control in distribution Networks" IEEE Bologna Power Tech Conference, Italy, June 23-26, 2003

[12] N.Iwan Santoso and Owen T.Tan,"Neural-Net Based Real time control of capacitors installed on distribution systems" IEEE Transactions on Power Delivery, Vol.5, No.1,January 1990

[13]Yuan-Yih Hsu and Feng-Chang Lu," A Combined artificial neural network-fuzzy dynamic programming approach to reactive power/ voltage control in a distribution substation" IEEE transactions on Power Systems, Vol.13, No.4, November 1998,pp.1265-1271

[14] K.S. Swaroop and P.S. Subhash," Neural network approach to voltage and reactive power control in power systems" IEEE conference, pp.228-233, 2005

\section{BIOGRAPHY}

P. N. Hrishikesh born in 1967 in India. He received B.E. degree from National Institute of Engineering, University of Mysore, Mysore, India in 1988 and M.Tech degree from National Institute of Engineering, Mysore, Visweswaraiah Technological University, India in the year 2000. He received Ph.D degree from Indian Institute of Technology Roorkee, India in 2010.

Currently he is working as a faculty in the Department of Electrical and Electronics Engg., Manipal Institute of technology, Manipal, India. He has published few papers in international and national conferences /journal. His research interest includes AI application to power system operation and control, distributed generation, embedded generation and distribution system automation

Jaydev Sharma received the B.E. degree from Jiwaji University, Gwalior, India, in 1968 and M.E. and Ph.D degrees from University of Roorkee, Roorkee, India, in the year 1971 and 1974 respectively.

Currently he is working as Professor in the Department of Electrical Engineering, Indian Instiute of Technology Roorkee, India. He has published more than 200 papers in reputed international and national journals/ conferences. $\mathrm{He}$ has supervised more than $30 \mathrm{Ph}$.d and more than 50 graduate theses. He has received many awards and honors. His research area of interest includes Power system planning, Power system operation and control, Distributed generation, AI applications to power system and distributed generation. 